\title{
Globular adiponectin induces differentiation and fusion of skeletal muscle cells
}

\author{
Tania Fiaschi ${ }^{1}$, Domenico Cirelli ${ }^{1}$, Giuseppina Comito ${ }^{1}$, Stefania Gelmini ${ }^{2}$, Giampietro Ramponi ${ }^{1}$, Mario Serio ${ }^{2}$, \\ Paola Chiarugi ${ }^{1}$ \\ ${ }^{I}$ Department of Biochemical Sciences, University of Florence, V.le Morgagni 50, 50134 Florence, Italy; ${ }^{2}$ Department of Clinic \\ Physiopathology, University of Florence, V.le Morgagni, 85, 50134 Firenze, Italy
}

The growing interest in skeletal muscle regeneration is associated with the opening of new therapeutic strategies for muscle injury after trauma, as well as several muscular degenerative pathologies, including dystrophies, muscular atrophy, and cachexia. Studies focused on the ability of extracellular factors to promote myogenesis are therefore highly promising. We now report that an adipocyte-derived factor, globular adiponectin (gAd), is able to induce muscle gene expression and cell differentiation. gAd, besides its well-known ability to regulate several metabolic functions in muscle, including glucose uptake and consumption and fatty acid catabolism, is able to block cell cycle entry of myoblasts, to induce the expression of specific skeletal muscle markers such as myosin heavy chain or caveolin-3, as well as to provoke cell fusion into multinucleated syncytia and, finally, muscle fibre formation. gAd exerts its prodifferentiative activity through redox-dependent activation of p38, Akt and 5'-AMP-activated protein kinase pathways. Interestingly, differentiating myoblasts are autocrine for adiponectin, and the mimicking of pro-inflammatory settings or exposure to oxidative stress strongly increases the production of the hormone from differentiating cells. These data suggest a novel function of adiponectin, directly coordinating the myogenic differentiation program and serving an autocrine function during skeletal myogenesis.

Keywords: globular adiponectin, reactive oxygen species, myogenesis

Cell Research (2009) 19:584-597. doi: 10.1038/cr.2009.39; published online 7 April 2009

\section{Introduction}

Adiponectin (also known as Acrp30) is an adipokine with antidiabetic and antiatherogenic functions. The hormone circulates in the plasma in associated structures (trimers, hexamers and high-molecular-weight forms) whose biological activities are poorly understood [1]. In the mammalian plasma both the full-length and globular forms of the hormone have been observed, although different organ targeting of the two adiponectin forms has

Correspondence: Paola Chiarugi

Tel: +39-055-4598343; Fax: +39-055-4598905

E-mail: paola.chiarugi@unifi.it

Abbreviations: gAd (globular adiponectin); DM (differentiation medium); mMHC (muscle myosin heavy chain); HOS (horse serum); ROS (reactive oxygen species)

Received 6 October 2008; revised 10 November 2008; accepted 25 November 2008; published online 7 April 2009 been suggested $[2,3]$. In particular, the activity of fulllength adiponectin seems to be restricted to the liver, while the globular form appears to be the main effector in the regulation of muscle glucose metabolism [4]. A decrease of plasma adiponectin content correlates with obesity, diabetes and insulin resistance, conditions that can be reversed in mice by treatment with exogenous adiponectin [5]. The physiological effects of the hormone on glucose and lipid metabolism are mediated by two receptors (AdipoR1 and AdipoR2) that have been identified and whose disruption abrogates adiponectin binding and metabolic actions $[6,7]$.

Adiponectin exerts its metabolic effects mainly in liver and skeletal muscle. In liver, adiponectin leads to reduced gluconeogenesis and increase of both glycogen synthesis and aerobic glucose consumption [8]. Recently, we reported that in hepatic cells the metabolic effects of globular adiponectin (gAd) are linked to a transient burst of reactive oxygen species (ROS). This oxidant produc- 
tion leads to a ligand-independent trans-activation of the insulin receptor and mediates the intracellular signalling and metabolic effects of the gAd in liver [9].

In muscle, gAd has been correlated to fatty acid oxidation, glucose uptake and lactate production [10]. While the metabolic action of the hormone has been full characterized, its intracellular pathways have been only partially elucidated. Recently, the adaptor protein APPL1 has been reported as an essential molecule for the downstream signalling cascade of the hormone both in skeletal muscle and in liver [11]. It has been reported that activation of 5'-AMP-activated protein kinase (AMPK) is essential for the metabolic effect of adiponectin in muscle, including glucose uptake, lactate production and inhibition of acetyl-coenzyme A-carboxylase [10]. In liver AMPK has been associated with decrease of gluconeogenesis enzymes by adiponectin [4].

Several data have indicated that in vivo adiponectin increases insulin sensitivity in both liver and skeletal muscle and ameliorates metabolic syndrome and diabetes [12]. Nevertheless, no studies have addressed whether adiponectin may act directly on muscle differentiation and regeneration. Owing to the insulin-like effects exerted by adiponectin in both liver and muscle, and considering that insulin treatment of myoblasts induces alignment and fusion of multinucleated myotubes, we investigated the involvement of adiponectin in muscle differentiation.

Myogenic differentiation is a complex event that involves morphological, biochemical and molecular modifications. Initially, pluripotent mesodermal cells give rise to myoblasts, which subsequently withdraw from the cell cycle and differentiate into multinucleated myotubes, expressing a range of muscle-specific proteins [13]. Several extracellular factors, such as hormones and growth factors (GFs), can modulate myogenic proliferation and differentiation. Hepatocyte GF, platelet-derived GF, fibroblast GF, insulin-like GF I and II, and insulin are able to control myogenesis during development and muscle regeneration $[14,15]$. In particular, the insulin-like GFs I and II, as well as insulin, potently stimulate myogenesis in vitro [16-18], and their presence is required for the development of skeletal muscle in vivo [19, 20]. Finally, ghrelin and sphingosine 1-phosphate have been recently reported to promote skeletal muscle differentiation and fusion of multinucleated myotubes [21, 22].

Here, we report that gAd is able to induce skeletal muscle differentiation in $\mathrm{C} 2 \mathrm{C} 12$ murine myoblasts. There is an adiponectin autocrine loop in differentiating myoblasts, leading to a clear increase in muscle lineage markers through a redox-dependent circuitry involving p38, Akt and AMPK signalling.

\section{Results}

gAd induces differentiation of C2C12 myoblasts

Chronic treatment with insulin produces alignment, elongation and fusion of myoblasts into multinucleated myotubes [19]. Owing to the wide insulin-mimetic effects exerted by adiponectin both in liver and in muscle, we were interested in testing the ability of the hormone to induce myogenesis in vitro. To this end we choose C2C12 myoblasts, a commonly used in vitro model for myogenesis. Growing myoblasts were treated for $72 \mathrm{~h}$ with $1 \mu \mathrm{g} / \mathrm{ml}$ gAd and in parallel with the common differentiation medium (DM) containing $2 \%$ horse serum (HOS). We observed that persistent treatment with gAd drove myoblasts towards muscle gene expression and cell differentiation (Figure 1A). Formation of multinucleated myotube, positive for the expression of muscle myosin heavy chain (mMHC), has been revealed by confocal analysis (Figure 1B). Cells treated with gAd showed the formation of multinucleated myotubes and a phenotype very similar to those treated with DM. The extent of differentiation induced by gAd in myoblasts was calculated through differentiation and fusion indexes, representing the degree of $\mathrm{mMHC}$ expression and the formation of multinucleated myotubes, respectively. As reported in Figure 1C and 1D, gAd-treated cells showed a significant increase of both differentiation and fusion indexes with respect to control, although the treatment with standard $\mathrm{DM}$ is much more effective.

In order to evaluate the ability of gAd to elicit the expression of muscle lineage differentiation markers, we analysed the mMHC and caveolin-3 content. Immunoblot analysis of 72-h-treated myoblasts revealed that gAd greatly increased the expression of both muscle-specific markers (Figure 2A). In particular, we observed that gAd-treated cells showed about 12-fold higher mMHC and caveolin-3 expression with respect to control cells. These findings suggest that gAd is able to elicit a response very similar to standard DM, being able to regulate the expression of skeletal muscle markers.

Proliferating $\mathrm{C} 2 \mathrm{C} 12$ myoblasts exit the cell cycle upon GF withdrawal and treatment with DM, to initiate irreversible differentiation. Interestingly, gAd is able to synergize with HOS to elicit skeletal muscle differentiation, as well as to antagonize the inhibitory activity of serum on differentiation, as indicated by the expression of mMHC (Figure 2B). Furthermore, we observed that 72-h treatment of myoblasts with gAd led to an increase in the cyclin-dependent kinase inhibitor p21. Again, gAd activity synergizes with HOS for inducing the accumulation of p21 kinase inhibitor (Figure 2C). These findings 

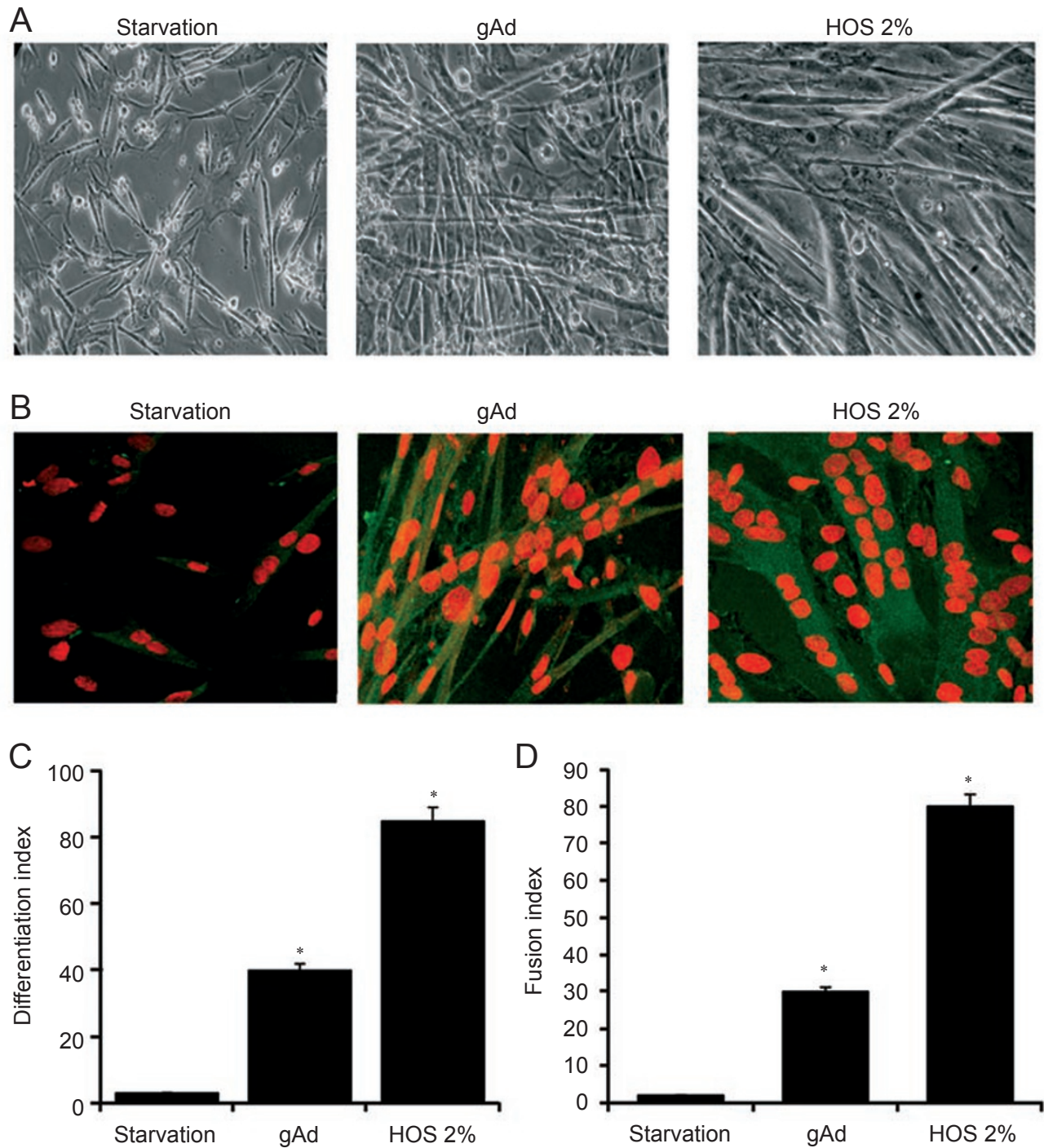

Figure $1 \mathrm{gAd}$ promotes myogenic differentiation of $\mathrm{C} 2 \mathrm{C} 12$ myoblasts. $\mathrm{C} 2 \mathrm{C} 12$ myoblasts (about $80 \%$ of confluence) were treated for $72 \mathrm{~h}$ with free-serum medium (starvation) with or without gAd $(1 \mu \mathrm{g} / \mathrm{ml}$ ) or with HOS $2 \%$. (A) Representative photographs of C2C12 myoblasts after $72 \mathrm{~h}$ of treatment with free-serum medium (starvation), with gAd or HOS $2 \%$. (B) Representative immunofluorescence of $\mathrm{C} 2 \mathrm{C} 12$ cells after different treatments. $\mathrm{C} 2 \mathrm{C} 12$ myoblasts were treated for $72 \mathrm{~h}$ as described in (A) and immunocytochemistry was performed. mMHC detection was checked using Alexa 488 fluorescent secondary antibodies (green), while nuclei were labelled with propidium iodide (red). (C and D) Differentiation index and fusion index in cells treated as described in (A) and calculated as reported in Materials and methods. C2C12 cells differentiated with HOS $2 \%$ were considered as positive control. All data are representative of at least three independent experiments. $* P<0.001$ vs starvation.

confirm that, similar to the effect of several differentiating agents, during myogenesis gAd is able to directly block the cell cycle, an indispensable feature of muscle differentiation.

Taken together, these data indicate that sustained treatment of $\mathrm{C} 2 \mathrm{C} 12$ cells with the adipokine gAd leads myoblasts towards a myogenic lineage, as observed by morphological analysis and the expression of skeletal muscle-specific markers.
gAd induces muscle differentiation through the activation of Akt, $p 38$ and AMPK pathways

To analyse the intracellular pathways activated during gAd-induced differentiation, we focused on p38 and Akt serine-threonine kinases, which have already been shown to be involved in myogenesis [23]. Figures $3 \mathrm{~A}$ and $3 \mathrm{~B}$ show that treatment of $\mathrm{C} 2 \mathrm{C} 12$ myoblasts for $72 \mathrm{~h}$ with gAd led to a strong activation of both Akt and p38 kinases (five- and twofold, respectively). Activation of these 
A
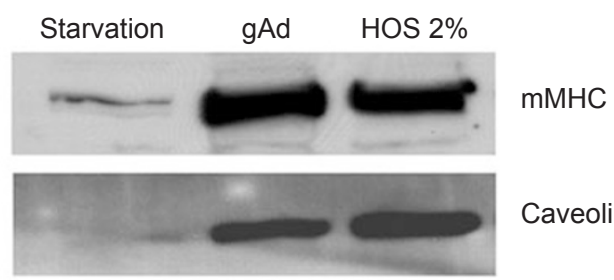

Caveolin-3

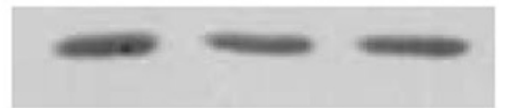

Actin
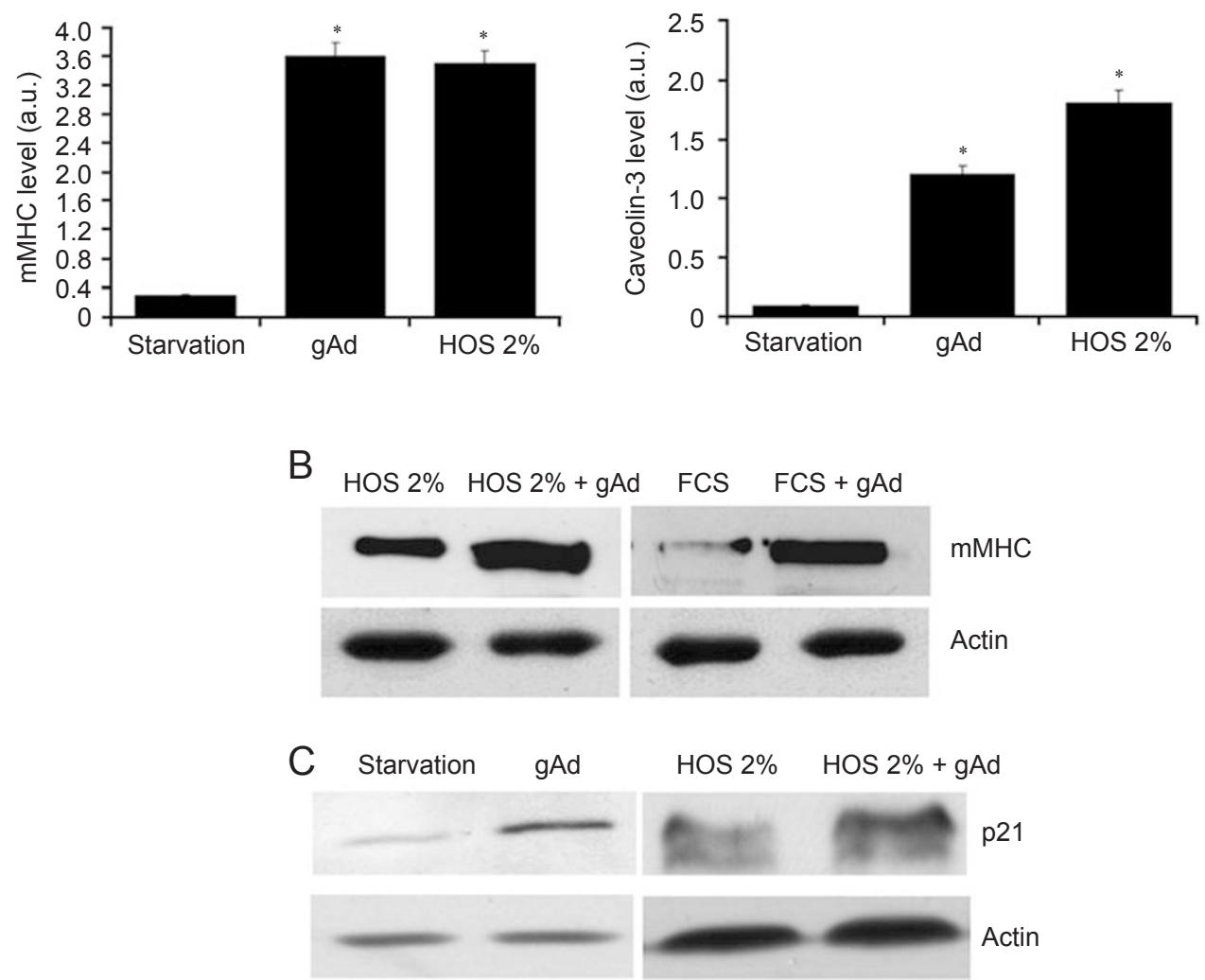

Figure $2 \mathrm{gAd}$ induces the expression of muscle differentiation markers. C2C12 myoblasts were treated for $72 \mathrm{~h}$ with HOS $2 \%$ or with free-serum medium (starvation) with or without $\mathrm{gAd}(1 \mu \mathrm{g} / \mathrm{ml})$. (A) The contents of mMHC and caveolin-3 were measured by western blots of whole-cell lysates and the intensity of the bands was quantified by densitometric analysis. (B) $\mathrm{C} 2 \mathrm{C} 12$ myoblasts were treated for $72 \mathrm{~h}$ with $\mathrm{HOS} 2 \%$ or with FCS $10 \%$ with or without $\mathrm{gAd}(1 \mu \mathrm{g} / \mathrm{ml})$. $\mathrm{mMHC}$ level was detected by western blot analysis of total cell lysates. (C) Murine myoblasts C2C12 were cultured for $72 \mathrm{~h}$ in free-serum medium (starvation) or HOS $2 \%$ with or without gAd $(1 \mu \mathrm{g} / \mathrm{ml})$. Western blot analysis of total cell lysates was performed to detect the expression of p21. All the western blots are representative experiments, while the bar graphs show the mean of four independent experiments. ${ }^{*} P<0.001$ vs starvation.

pathways is essential for the induction of myogenesis by gAd. Indeed, inhibition of the p38 or the phosphatidylinositol 3-kinase/Akt pathway abolishes the effect of gAd in inducing myoblast differentiation. We used SB203580 to inhibit p38 kinase and LY294002 to block PI3K, the upstream regulator of Akt. Inhibition of either pathway greatly affected the formation of myotubes (Figure 3C) and the expression of mMHC (Figure 3D).

Besides Akt and p38, we analysed the role of AMPK in gAd-induced myogenesis. Although AMPK has already been reported to play a key role in adiponectin signalling for glucose and lipid metabolism in both liver and muscle, its involvement in myogenesis has not been investigated. We observed that gAd was able to induce a 
A

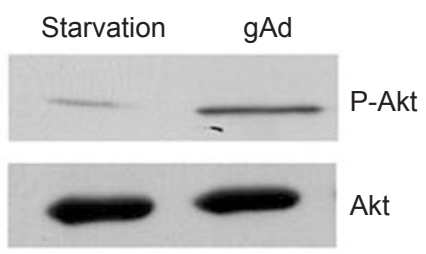

B

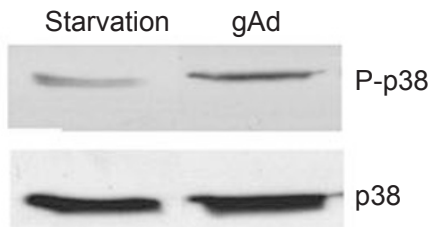

C

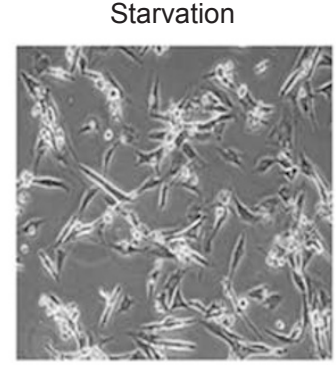

gAd

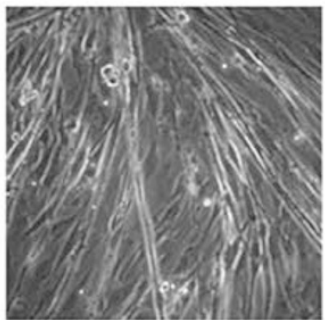

gAd + LY294002

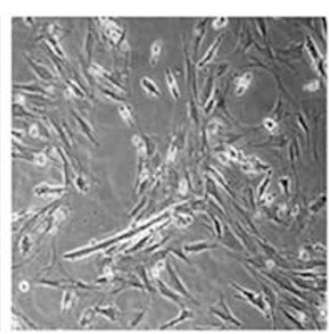

gAd + SB203580

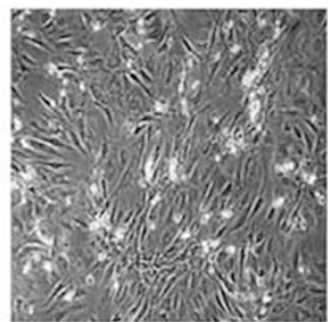

D

$\mathrm{gAd}+\mathrm{gAd}+$

Starvation gAd LY294002 SB203580
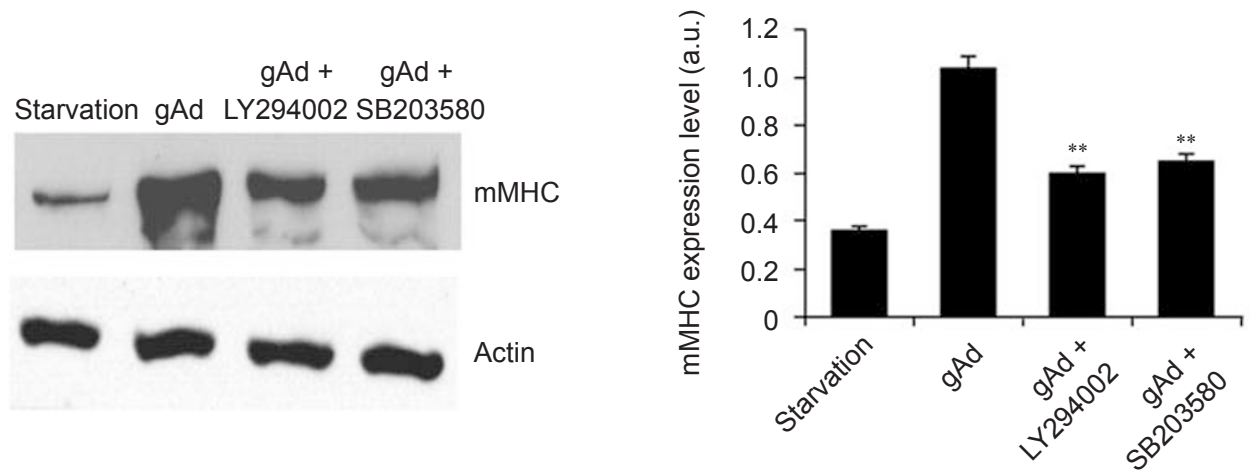

Figure 3 Analysis of the signalling pathways involved in gAd-induced myogenesis. (A and B) Activation of Akt and p38 by chronic treatment of $\mathrm{C} 2 \mathrm{C} 12$ cells with gAd. $\mathrm{C} 2 \mathrm{C} 12$ myoblasts were cultured for $72 \mathrm{~h}$ in free-serum medium (starvation) with or without gAd $(1 \mu \mathrm{g} / \mathrm{ml})$. Total-cell lysates were used in western blot analysis to detect the phosphorylation level of Akt (Ser-473) and p38 (Thr180/Tyr182). (C) C2C12 cells were pre-treated for 30 min with LY294002 (10 $\mu$ M) or with SB203580 (5 $\mu$ M) before adding free-serum medium (starvation) with or without $\mathrm{gAd}(1 \mu \mathrm{g} / \mathrm{ml})$ for $72 \mathrm{~h}$. A representative photograph of differently treated cells is shown in (C). (D) The expression level of mMHC was detected on total-cell lysates of treated cells by western blot analysis, and the bands were then quantified by densitometric analysis. All the data are representative of different experiments and the bar graphs show the means of the three different experiments. $* P<0.001$ vs starvation, ${ }^{* *} P<0.001$ vs gAd.

fourfold activation of AMPK in 72-h-treated myoblasts (Figure 4A). To analyse the role of AMPK activation in myogenesis, we used a synthetic activator of the kinase, 5-aminoimidazole-4-carboxamide-1-beta-4-ribofuranoside (AICAR). Treatment of myoblasts with AICAR produced an effect very similar to gAd administration, 
A

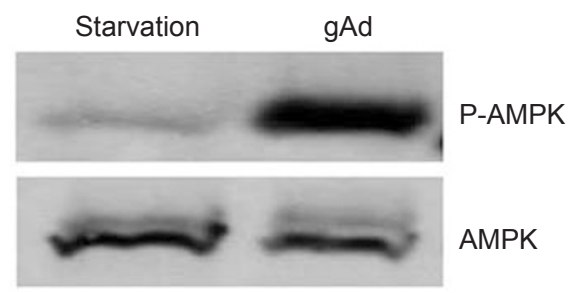

B
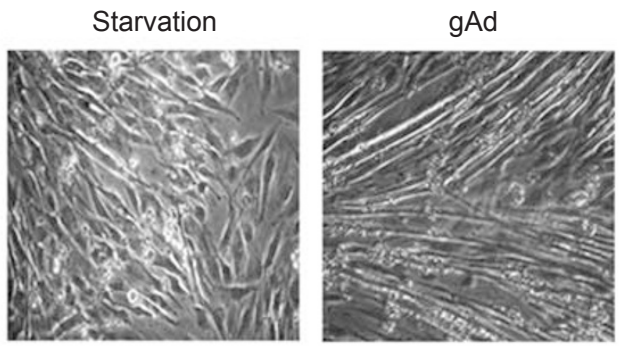

C

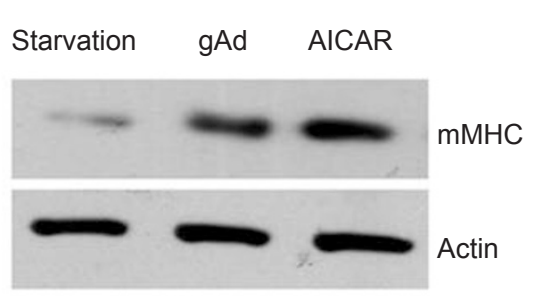

gAd +

Starvation gAd Compound C

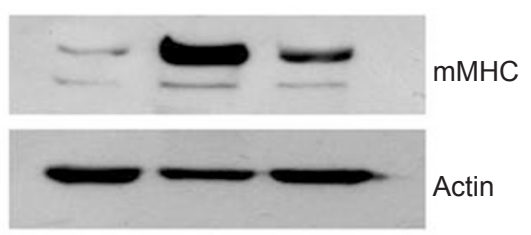

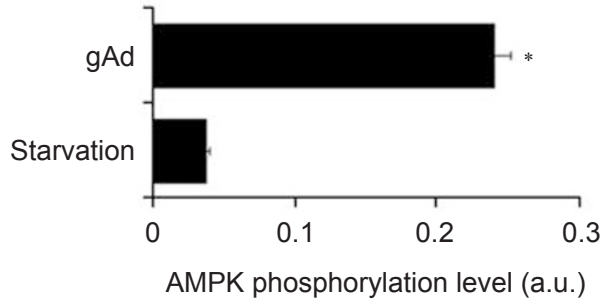

AICAR

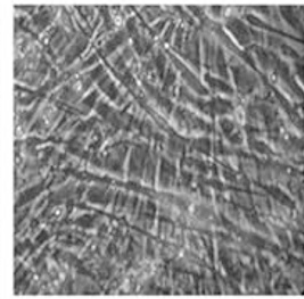

gAd + Compound C
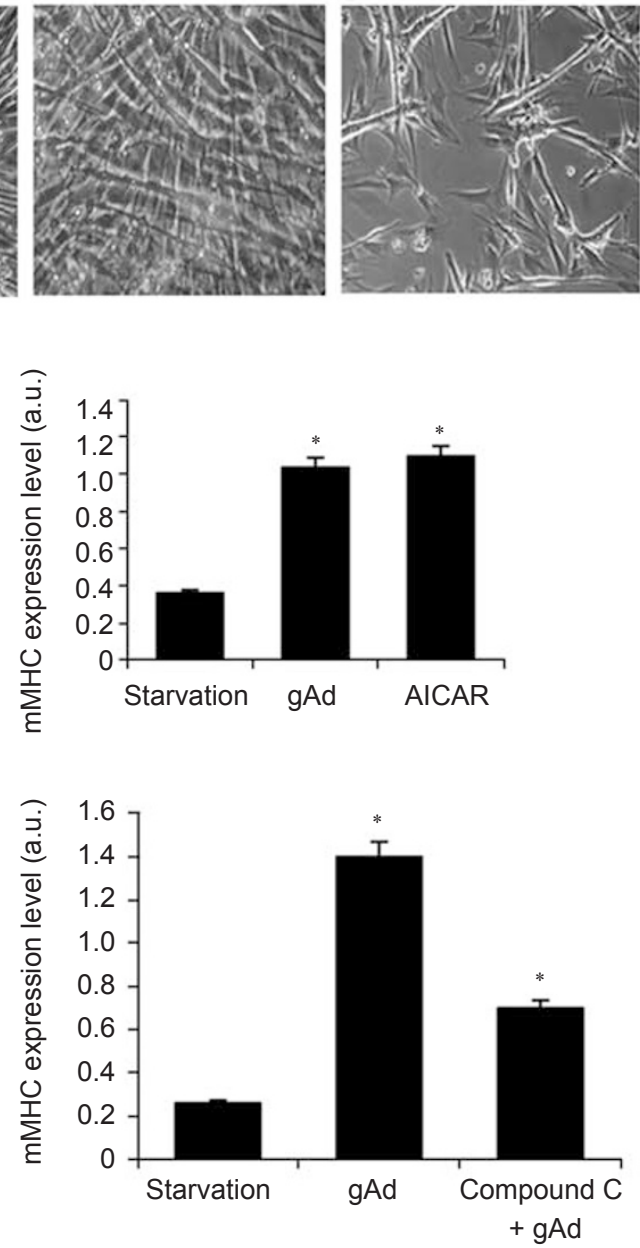

Figure 4 AMPK activation is involved in gAd-induced myogenesis. (A) C2C12 cells were treated for $72 \mathrm{~h}$ with free-serum medium (starvation) with or without $\mathrm{gAd}(1 \mu \mathrm{g} / \mathrm{ml})$. The activation of AMPK was checked on total cell lysates using antibodies against phospho-Thr471 of AMPK. The bands were then quantified by densitometric analysis. (B) Representative photographs of C2C12 myoblasts after $72 \mathrm{~h}$ of treatment with free-serum medium (starvation) with or without $\mathrm{gAd}(1 \mu \mathrm{g} / \mathrm{ml})$ or with free-serum medium (starvation) containing AICAR (2 mM). (C) Total cell lysates obtained from murine-treated-myoblasts described in (B) are used to detect the level of expression of mMHC. (D) C2C12 cells were treated for 45 min with Compound $\mathrm{C}(20 \mu \mathrm{M})$ before adding freeserum medium with or without $\mathrm{gAd}(1 \mu \mathrm{g} / \mathrm{ml})$ for $72 \mathrm{~h}$. The western blots are representative of four different experiments. The bar graph represents the mean of the values obtained in four independent experiments. ${ }^{*} P<0.001$ vs starvation.

as indicated by both myotube formation (Figure 4B) and mMHC expression (Figure 4C). In addition, the role of AMPK in induction of myogenesis by gAd was confirmed by the use of the AMPK inhibitor Compound $\mathrm{C}$, which greatly affected the expression of mMHC (Figure 4D).

Collectively, these observations demonstrate that in myoblasts activation of the p38, Akt and AMPK pathways by gAd is a key event in the ability of the adipokine 
to induce commitment of muscle precursors towards differentiation.

gAd induces myogenesis in a redox-dependent manner

Recently we demonstrated that gAd leads to a transient increase in ROS in liver. ROS are key mediators of the metabolic effects exerted by the hormone and are responsible for the ligand-independent trans-activation of insulin receptor [9]. Based on these previous observations, we analysed the involvement of ROS in the induction of myogenesis by gAd. In contrast with the action of gAd in the liver, in muscle cells we did not observe any trans-phosphorylation/activation of the insulin receptor in response to gAd administration (data not shown). Nevertheless, elimination of redox signalling elicited by gAd through chronic treatment of gAd-treated differen-
A

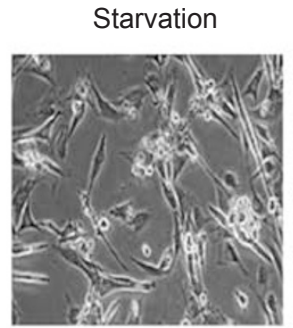

B

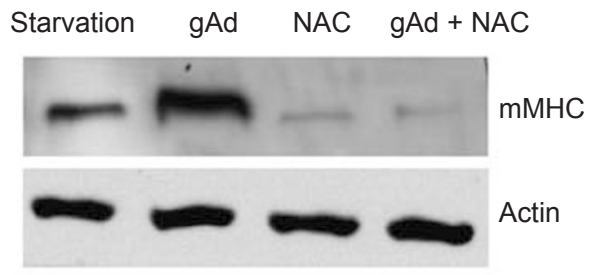

gAd
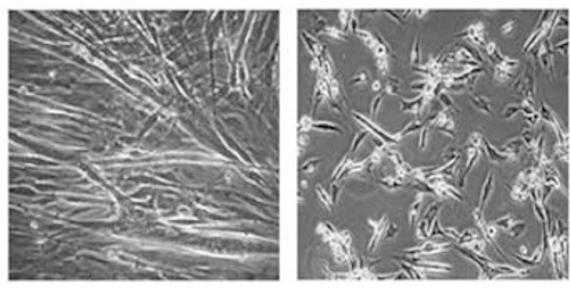

$\mathrm{gAd}+\mathrm{NAC}$

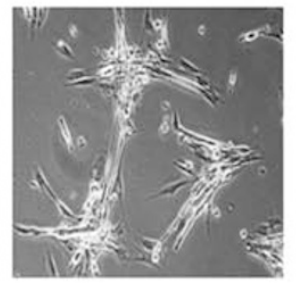

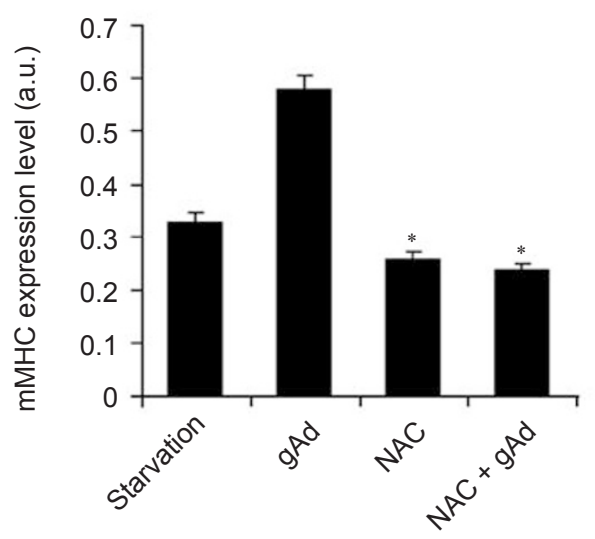

C

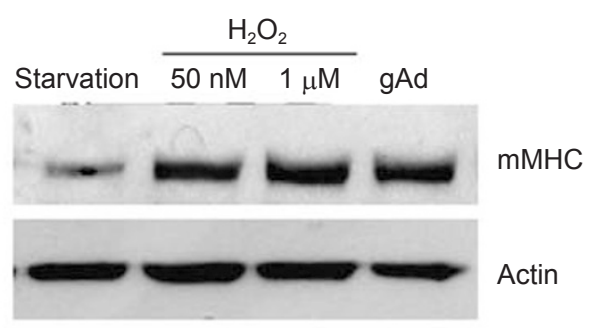

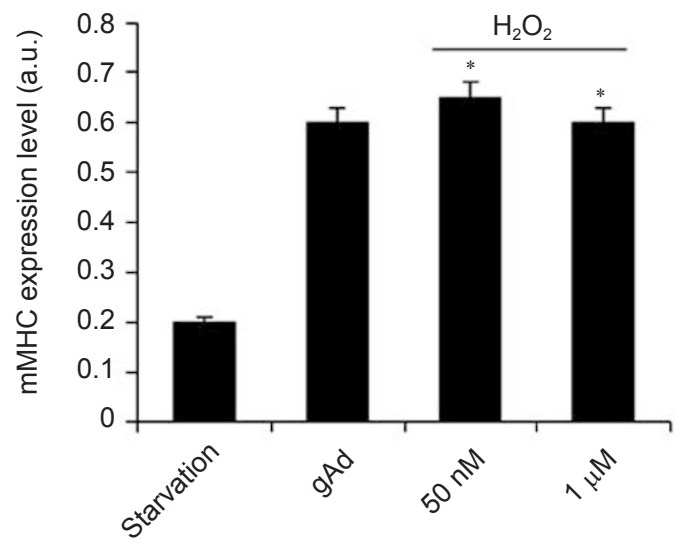

Figure 5 gAd-induced myogenesis is redox-dependent. (A) Representative photograph of $\mathrm{C} 2 \mathrm{C} 12$ myoblasts after $72 \mathrm{~h}$ of treatment with free-serum medium (starvation) with or without gAd $(1 \mu \mathrm{g} / \mathrm{ml})$. Where indicated cells were pre-treated with NAC $(10 \mathrm{mM})$ for $30 \mathrm{~min}$ before adding gAd. (B) Total cell lysates of cells described in (A) were used in a western blot analysis to detect the level of expression of $\mathrm{mMHC}$. (C) $\mathrm{C} 2 \mathrm{C} 12$ were treated as described in (A), except for the addition of $\mathrm{H}_{2} \mathrm{O}_{2}$ where indicated. The blots show a representative experiment, while the bar graph corresponds to the mean of three independent experiments. $* P<0.001$ vs starvation. 
A

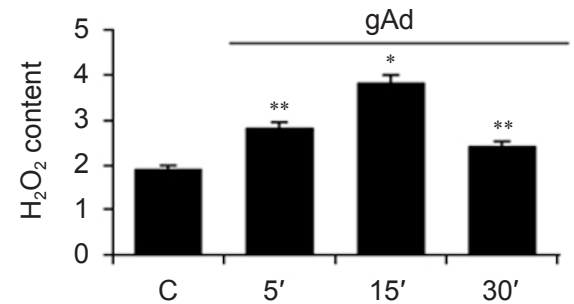

B
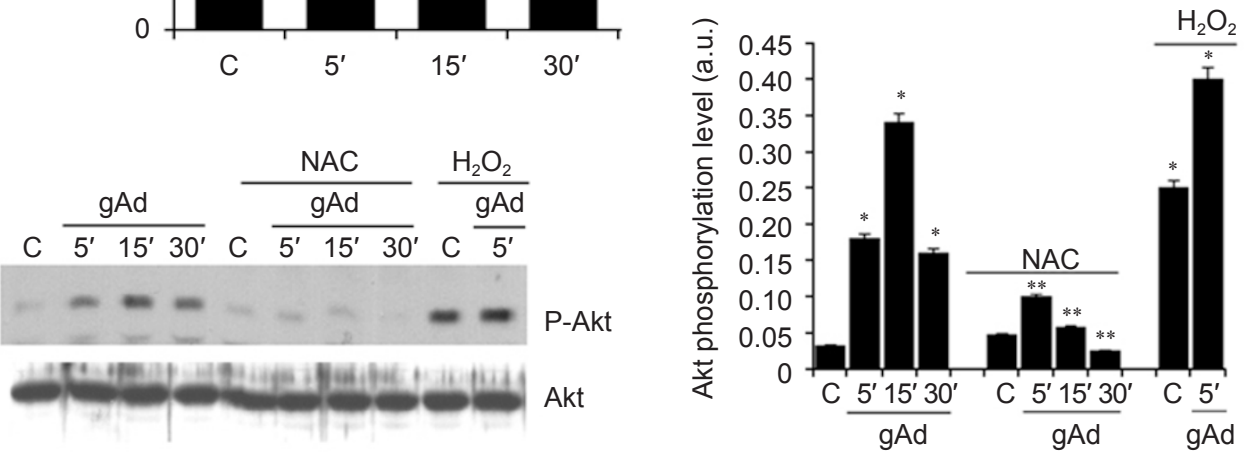

C
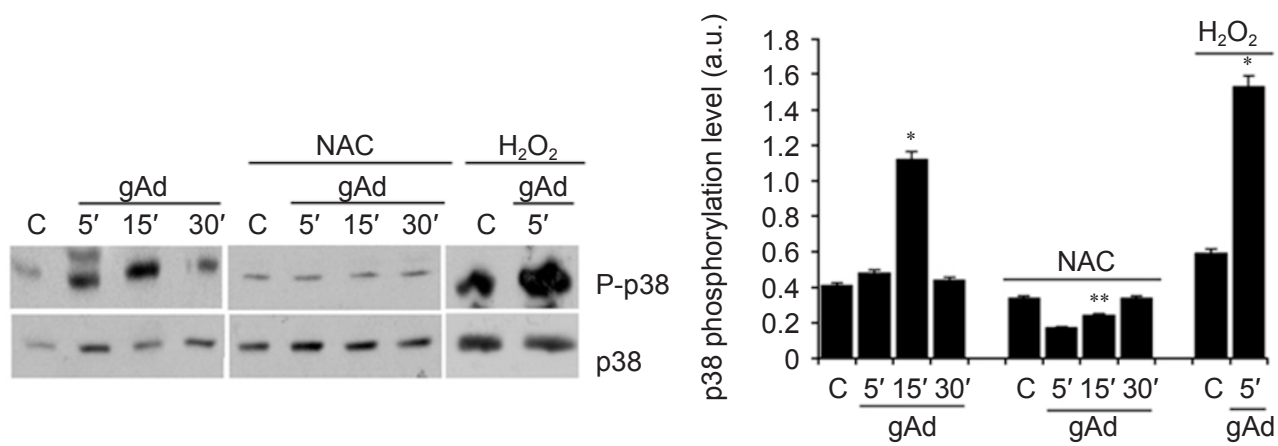

D

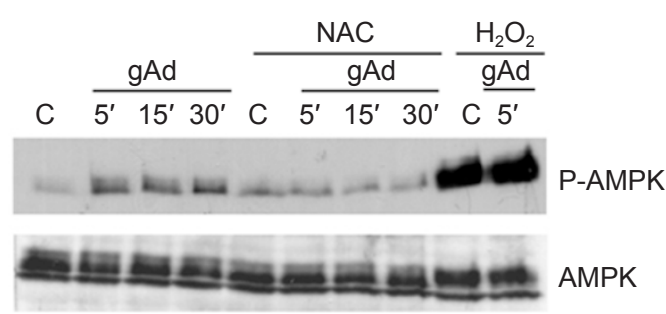

E
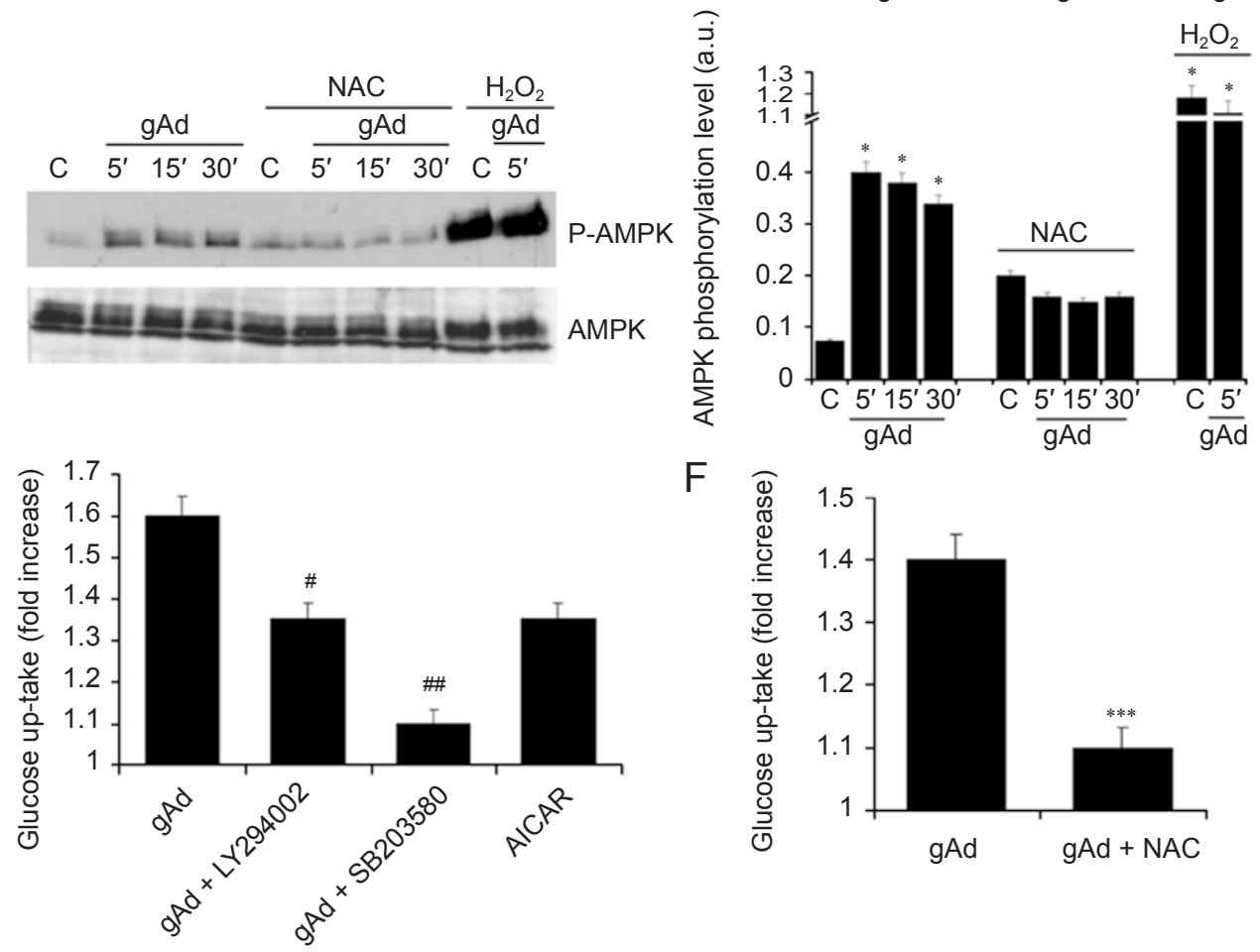

$\mathrm{F}$

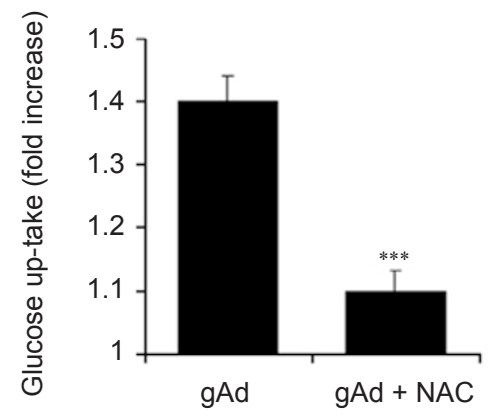


tiating cells with the ROS scavenger $N$-acetyl-cysteine (NAC) strongly impairs muscle differentiation. Indeed, NAC administration blocked both myotube formation (Figure 5A) and mMHC expression (Figure 5B), thus suggesting that the ability of gAd to induce myogenesis is strongly redox-dependent. In addition, treatment of C2C12 myoblasts with $\mathrm{H}_{2} \mathrm{O}_{2}$ for $72 \mathrm{~h}$ led to the expression of $\mathrm{mMHC}$, indicating the positive role of ROS in muscle differentiation (Figure 5C).

Next, we observed that consistent with its effect in the liver, gAd induced an increase in ROS in differentiating $\mathrm{C} 2 \mathrm{C} 12$ cells (Figure 6A). To analyse the role of these oxidant molecules in the myogenesis process, we treated differentiating cells with $20 \mathrm{mM}$ NAC or $100 \mu \mathrm{M}$ hydrogen peroxide in concomitance with $\mathrm{gAd}$, and evaluation of the p38, Akt and AMPK pathways was carried out. Figure 6 shows that removal of ROS by NAC almost completely abolished the gAd-induced activation of the three kinases, while the administration of exogenous hydrogen peroxide was able to strongly mimic gAd treatment. Interestingly, while for p38 and Akt activation a synergistic effect of hydrogen peroxide and the adipokine was observed, AMPK was fully activated by hydrogen peroxide and gAd did not cause a further increase (Figure 6B-6D).

gAd plays a key role in skeletal muscle metabolism, mainly through the up-regulation of glucose uptake, thus contributing to lowering, in synergy with insulin and blood glucose level [4, 10, 24]. Interestingly, in mouse muscle the main pathways leading to glucose uptake are indeed mediated by p38, Akt and AMPK $[6,10,25]$. We first observed that in differentiating myotubes gAd induced a $60 \%$ increase in glucose uptake (Figure 6E). This increase was strongly sensitive to p38 and Akt inhibition, although the contribution of the two pathways appears to be different. Interestingly, administration of AICAR, the synthetic activator of AMPK, was able to partly mimic the increase in glucose uptake elicited by gAd. These findings indicate that the increase in glucose uptake induced by gAd is likely mediated by the synergistic action of p38, Akt and AMPK pathways. We further checked whether the upregulation of glucose intracellular transport elicited by gAd in differentiating myotubes is linked to the generation of ROS. We observed that removal of ROS by treatment with $20 \mathrm{mM}$ NAC fully abolished the effect of gAd in inducing the intracellular accumulation of glucose (Figure 6F).

These findings demonstrate that gAd signalling in differentiating myotubes is able to control skeletal muscle glucose uptake through a complex redox circuitry involving $\mathrm{p} 38$, Akt and AMPK pathways.

\section{Myogenesis in response to adiponectin signalling in-} volves an autocrine loop

Adiponectin is mainly secreted by adipose tissue, although recently pro-inflammatory cues have been indicated as responsible for the release of adipokine from endothelial and/or muscle cells [26]. We therefore checked the ability of differentiating $\mathrm{C} 2 \mathrm{C} 12$ cells to directly produce adiponectin. 72-h HOS-differentiated cells were assayed by anti-adiponectin immunoblot (Figure 7A). Differentiating cells exposed to chronic treatment with the pro-inflammatory cytokines tumour necrosis factor- $\alpha$ $(\mathrm{TNF} \alpha)$ and interferon- $\gamma(\mathrm{INF} \gamma)$ were used as control. The results reveal that during myogenesis $\mathrm{C} 2 \mathrm{C} 12$ cells are autocrine for the production of adiponectin, although the production of the hormone can be further increased by a concomitant pro-inflammatory cue. In addition, chronic treatment of differentiating muscle cells with $100 \mu \mathrm{M}$ hydrogen peroxide reveals that a condition of exogenous oxidative stress is able to increase the production of adiponectin as well.

To analyse whether myogenesis is able to modulate the responsiveness of differentiating muscle cells to adi-

Figure $6 \mathrm{gAd}$ signalling in differentiating myoblasts is redox-dependent. Murine myoblasts $\mathrm{C} 2 \mathrm{C} 12$ were differentiated for 3 days with HOS $2 \%$ and then serum-starved for $24 \mathrm{~h}$. Cells were stimulated with $\mathrm{gAd}(1 \mu \mathrm{g} / \mathrm{ml})$ for the indicated period. (A) gAd stimulation induces a transient production of hydrogen peroxide in differentiating myoblasts. Intracellular production of $\mathrm{H}_{2} \mathrm{O}_{2}$ was assayed as described previously [9]. $* P<0.001$ and $* * P<0.05$ vs control. (B-D) Cells were treated as described in (A). Where indicated, cells were treated for 30 min before the stimulation with NAC 20 mM or with $\mathrm{H}_{2} \mathrm{O}_{2}(100 \mu M)$ exogenously added to the cells. Total-cell lysates were used to detect the phosphorylation of Akt (Ser-473) (B) the activation of p38 (Thr180/Tyr182) (C) and the phosphorylation of AMPK (Thr-471) (D). Immunoblots are representative of three different experiments. The bar graphs show the mean of the ratio of phosphorylated bands $v s$ total protein. $* P<0.001$ vs control of untreated cells, ${ }^{* *} P<0.001$ vs the corresponding time of stimulation of gAd-stimulated cells. (D) gAd-induced glucose uptake in myotubes are p38-, Akt- and AMPK-dependent. Before stimulation with gAd, 3-days-differentiating C2C12 cells were pretreated for 30 min with LY294002 $(10 \mu \mathrm{M})$ or with SB203580 $(5 \mu \mathrm{M})$ or with AICAR $(2 \mathrm{mM}) .{ }^{\#} P<0.01 \mathrm{vs}$ gAd; ${ }^{\# \#} P<0.001 \mathrm{vs}$ gAd. (E) gAd-induced glucose uptake is redox-dependent. Before stimulation with gAd, 3-days-differentiating C2C12 cells were pre-treated for $30 \mathrm{~min}$ with NAC $(20 \mathrm{mM})$. The values are presented as fold increase with respect to control and correspond to the mean of three independent experiments. $* * * P<0.001$ vs gAd. 
A

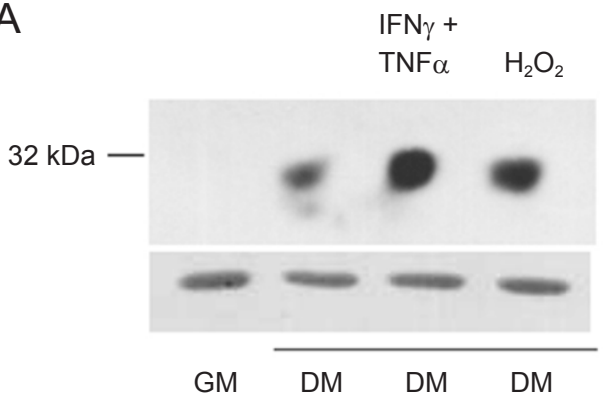

Ip: $\alpha$ Acrp30

Wb: $\alpha$ Acrp30

Wb: $\alpha$ actin

B

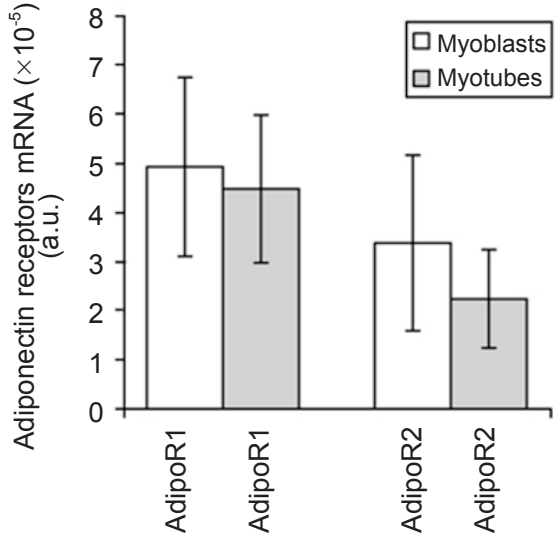

C

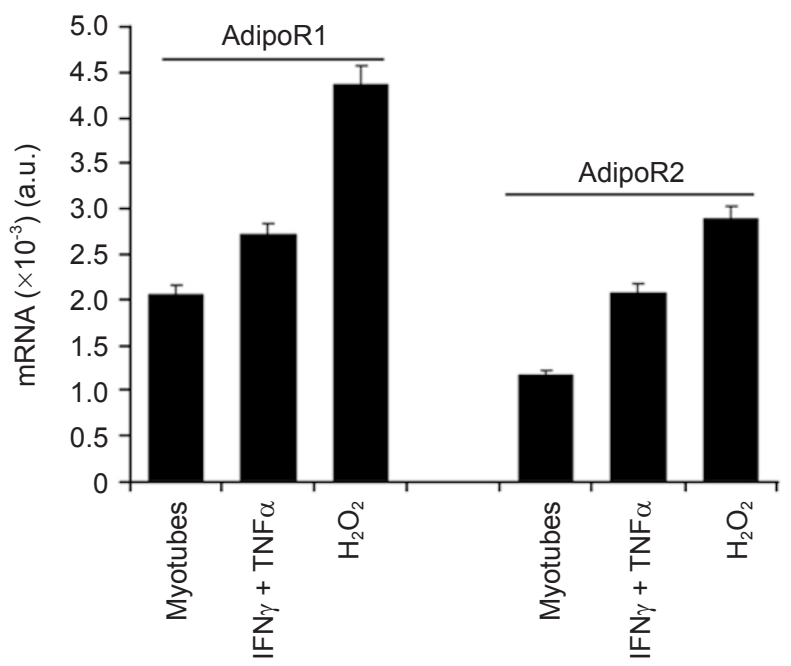

Figure 7 Myotubes are autocrine for adiponectin production. (A) Adiponectin production was assayed in myoblasts and in 3-days-differentiating myotubes. Where indicated differentiating myotubes (after 3 days of culture in HOS 2\%) were treated for $48 \mathrm{~h}$ with IFN $\gamma(10 \mathrm{ng} / \mathrm{ml})$ and TNF $\alpha(10 \mathrm{ng} / \mathrm{ml})$ or with $\mathrm{H}_{2} \mathrm{O}_{2}$ $(100 \mu \mathrm{M})$. (B) Adiponectin receptor expression in myoblasts and in 3-days-differentiating myoblasts. (C) 3-days-differentiating myoblasts are treated for $48 \mathrm{~h}$ with IFN $\gamma(10 \mathrm{ng} / \mathrm{ml})$ and TNF $\alpha$ (10 ng/ml) or with $\mathrm{H}_{2} \mathrm{O}_{2}(100 \mu \mathrm{M})$. In (B) and (C), expressions of AdipoR1 and AdipoR2 are obtained by real-time PCR as described in Materials and methods. Adiponectin receptor mRNA is expressed as $2^{-\Delta \Lambda C T}$. Results (means $\pm \mathrm{SD}$ ) are the means of three different experiments. ponectin, we analysed by real-time PCR the expression of its receptors AdipoR1 and AdipoR2. AdipoR2 shows a strict restriction to liver, while AdipoR1 is widely distributed and is predominant in skeletal muscle $[4,6]$. Our results indicate that myogenesis does not significantly affect the level of messengers of both AdipoR1 and AdipoR2 receptors (Figure 7B). In contrast, mimic of a proinflammatory condition by concomitant treatment with TNF $\alpha$ and INF $\gamma$ induced an increase of both AdipoR1 and AdipoR2 mRNAs, and a more pronounced increase was observed following the exogenous addition of $100 \mu \mathrm{M}$ hydrogen peroxide (Figure 7C).

Taken together, these data indicate that during skeletal muscle differentiation myotubes represent an autocrine system for adiponectin production, whose amount may be further increased by pro-oxidant/inflammatory conditions, leading to increased responsiveness to the hormone.

\section{Discussion}

In this paper, we report for the first time that in myoblasts adiponectin is able to induce muscle gene expression and cell differentiation. The ability to induce differentiation depends on the activation of p38, Akt and AMPK pathways and involves a specific redox signalling culminating in increased expression of skeletal markers such as mMHC and caveolin-3. In addition, we report that differentiating myoblasts elicit a positive loop, which, through the autocrine production of adiponectin, might strengthen the activity of the hormone during de novo differentiation.

Upon muscle diseases or direct injury, quiescent satellite cells, located between the sarcolemma and the basal membrane of terminally differentiated adult muscle fibres, are activated to undergo proliferation and differentiate to allow muscle regeneration [27]. Skeletal muscle regeneration involves precursor cell proliferation, commitment to final differentiation, cell fusion into multinucleated syncytia and, finally, muscle fibre formation [14]. Mechanisms leading to muscle regeneration are poorly understood, and the known extracellular factors regulating such processes are very few. Now, besides some known factors regulating myogenesis, such as insulin, the insulin-like GFs, the fibroblast GFs, ghrelin and shingosine-1-phosphate, our data show that gAd also belongs to this group $[16,19$, $21,22,28,29]$.

Interestingly, gAd appears to be able to regulate several steps of myogenesis. Indeed, satellite cell differentiation into skeletal muscle follows a coordinated set of events, including (i) exit of the cell cycle and accumulation of the cyclin-dependent kinase inhibitor of the p21 family; (ii) expression of proteins of the muscle 
regulatory factor family, such as myogenin, or MyoD; (iii) commitment to irreversible differentiation; (iv) expression of muscle-specific proteins, such as mMHCs, myosin light chains and caveolin-3; and, finally, (v) fusion of myocytes into multinucleated myotubes [30]. We have herein reported that gAd is able to influence several of these events. Indeed, similar to standard DM-containing HOS, gAd treatment blocks the cell cycle through the accumulation of the cyclin kinase inhibitor $\mathrm{p} 21$, commits cells towards terminal differentiation, causing expression and accumulation of both early and late specific muscle lineage markers such as mMHC and caveolin-3, and, finally, brings forth fusion of myocytes into multinucleated syncytia. Hence, gAd is able to behave as a broad muscle differentiation factor, its action being not restricted to one particular event within the differentiation process.

Forced activation of $\mathrm{p} 38$ and Akt pathways in proliferating $\mathrm{C} 2 \mathrm{C} 12$ myoblasts is sufficient to induce expression of muscle markers, cell cycle exit and terminal differentiation [31, 32]. gAd has already been linked to activation of both p38 and Akt in muscle, and these events have been correlated with the induction of GLUT4 membrane translocation and the consequent increase in glucose uptake $[6,10,11]$. Thus, we investigated whether gAd pro-differentiative activity is mediated by p38 or Akt. First, we showed that gAd activates both p38 and Akt in $\mathrm{C} 2 \mathrm{C} 12$ cells. Consistently, inhibition of p38 or Akt by treatment with SB203580 or LY294002 resulted in almost complete inhibition of gAd-induced differentiation. Altogether, these data demonstrate that in muscle the effect elicited by gAd on p38 and Akt goes beyond the simple regulation of glucose entry in muscle cells, as these pathways are strongly involved in the orchestration of the antiproliferative and pro-differentiative activities of the adipokine.

Besides Akt and p38, AMPK is also involved in the ability of gAd to regulate myogenesis. AMPK is a central sensor of muscle energy requirements as it is activated by the high AMP/ATP ratio. gAd has already been indicated as a strong activator of the kinase, mainly inducing its phosphorylation in the activation loop. gAd-induced activation of AMPK is involved in inhibition of acetyl coenzyme-A carboxylase, and increase of fatty-acid combustion, glucose uptake, as well as lactate production in myocytes [33]. Besides these insulin-mimetic effects elicited by gAd and transduced by AMPK, we now show that $\mathrm{gAd} / \mathrm{AMPK}$ signalling is also involved in gAdinduced muscle differentiation.

We have recently reported that in liver cells several insulin-mimetic effects elicited by gAd, including glucose uptake and synthesis of glycogen upon hyperglycaemia, are mediated by a ligand-independent trans-activation of the insulin receptor itself. This outcome involves generation of ROS by gAd signalling, and elimination of redox signalling has a profound effect on both liver glucose consumption and storage upon gAd treatment [9]. Our present results indicate that, although in myoblasts gAd does not lead to ligand-independent activation of the insulin receptor (data not shown), ROS play a key role in gAd downstream signalling. Indeed, gAd elicits ROS increase in differentiating myoblasts, and elimination of ROS by scavenger administration abolishes the prodifferentiative effect of gAd. Elimination of ROS dramatically reduces activation of $\mathrm{p} 38$, Akt and AMPK in response to $\mathrm{gAd}$, while hydrogen peroxide mimics hormone treatment. Consistently, scavenging redox signalling in differentiating cells strongly interferes with gAdinduced glucose uptake. We therefore suggest that during myogenesis gAd redox signalling, although it does not induce direct activation of the insulin receptor, is a required step for the regulation of several downstream pathways, which, nevertheless, are involved in insulinmimetic outcomes.

Upon muscular injury, skeletal myoblasts are activated to terminally differentiate through an autocrine or a paracrine loop. We speculate that gAd might contribute to skeletal muscle plasticity, promoting the differentiation and fusion of myoblasts in the damaged muscles. Although gAd is a circulating hormone mainly secreted by the adipose tissue, there are a few indications for its extra-adipocyte synthesis, suggesting both endocrine and paracrine/autocrine effects. The autocrine production of adiponectin in endothelial cells has been correlated with antiatherogenic properties of the hormone. In addition, pro-inflammatory cues elicit in muscle an autocrine production of adiponectin [26]. Here we report that myogenesis induces an autocrine production of adiponectin, which can be further increased by treatment with proinflammatory cytokines or exposure to oxidative stress, thereby suggesting an autocrine-loop-sustaining myogenesis. Inflammation may play a key role in this autocrine loop for three different reasons. First, inflammation may be important for proteolytic generation of $\mathrm{gAd}$, as the lymphocyte-secreted elastase is involved in the generation of gAd from the full-length pro-hormone [34]. Second, inflammation is linked to an oxidative stress that, on the basis of our data, may contribute to strengthening the effect of the hormone on the regenerating muscle [35]. Last, inflammation has already been reported to synergize with other factors affecting muscle regeneration, mainly through the orchestration of a pro-activating and pro-differentiative microenvironment for muscle stem cells.

By showing that gAd stimulates terminal differentia- 
tion of skeletal myoblasts in vitro, we hypothesized that the hormone may be involved in skeletal muscle differentiation in vivo. A functional analysis in adiponectin knockout mice indicated a clear role of the hormone as an insulin-sensitizing adipokine, but reveals the lack of a consistent phenotype for embryonic myogenesis, suggesting that adiponectin function is not required for myogenesis during development [7]. However, although not essential for ontogenesis, adiponectin might be involved in adulthood myogenesis during the regenerative processes of skeletal muscle induced by trauma or degenerative diseases such as dystrophies, muscular atrophy and cachexia.

Our data may provide a novel paradigm that an adipocyte-derived hormone would contribute to skeletal muscle plasticity, promoting the differentiation and fusion of myoblasts in the damaged muscles. Reduced adiponectin levels are caused by interactions of genetic and environmental factors, causing obesity, insulin resistance and diabetes, the key characteristics of metabolic syndrome [4, 12]. Hence, a relationship between metabolic syndrome and muscle regenerative ability may be proposed. Although specific data about the impairment of muscle regeneration in patients with metabolic syndrome are lacking, we can speculate that agonist activation of gAd differentiative activity as well as overexpression of the hormone may provide novel therapeutic strategies for several skeletal muscle pathologies associated with impaired muscle regeneration.

\section{Materials and Methods}

\section{Reagents}

$\mathrm{C} 2 \mathrm{C} 12$ cells were a generous gift of Dr M Massimino from the University of Padua. Unless specified, all reagents were obtained from Sigma, except for PVDF membrane (Millipore), anti-AMPK, anti-Acrp30, anti-p21, anti-mMHC antibodies (Santa Cruz), antiphospho-p38, anti-p38 and anti-phospho-AMPK antibodies (Cell Signalling). gAd was from Alexis, and Alexa 488 fluorescent secondary antibodies and 2',7'-dichlorofluorescein diacetate (DCFDA) were from Molecular Probes.

\section{Cell cultures}

C2C12 cells were cultured in Dulbecco's modified Eagle's medium supplemented with $10 \%$ fetal bovine serum in $5 \% \mathrm{CO} 2$ humidified atmosphere. For differentiation, sub-confluent $\mathrm{C} 2 \mathrm{C} 12$ were shifted from growing medium to DM composed of Dulbecco's modified Eagle's medium containing $2 \%$ HOS or gAd $1 \mu \mathrm{g} / \mathrm{ml}$.

\section{Immunoprecipitation and western blot analysis \\ $1 \times 10^{6}$ cells were lysed for $20 \mathrm{~min}$ on ice in $500 \mu \mathrm{l}$ of RIPA lysis buffer $(0.1 \%$ SDS, $0.5 \%$ deoxycholate, $50 \mathrm{mM}$ Tris- $\mathrm{HCl}, \mathrm{pH}$ 7.5, $150 \mathrm{mM} \mathrm{NaCl}, 1 \%$ Nonidet P-40, 2 mM EGTA, 1 mM sodium orthovanadate, $1 \mathrm{mM}$ phenyl-methanesulphonyl-fluoride, $10 \mu \mathrm{g}$ / $\mathrm{ml}$ aprotinin, $10 \mu \mathrm{g} / \mathrm{ml}$ leupeptin). Lysates were then clarified by}

centrifugation. Immunoprecipitation was performed for $4 \mathrm{~h}$ at $4{ }^{\circ} \mathrm{C}$ with 1-2 $\mu \mathrm{g}$ of the specific antibodies. Immune complexes were collected on protein A-Sepharose, separated by SDS/PAGE, and transferred onto PVDF. Immunoblots were obtained as described previously [9] and analysed by a Biorad ChemiDoc-It Imaging System for dedicated chemiluminescent image acquisition.

\section{Immunofluorescence}

$6 \times 10^{4} \mathrm{C} 2 \mathrm{C} 12$ cells were grown on glass coverslips in growing medium. After $24 \mathrm{~h}$, in order to induce differentiation, C2C12 cells were treated with free-serum medium (starvation), with or without gAd $1 \mu \mathrm{g} / \mathrm{ml}$, or DM for $72 \mathrm{~h}$. Cells were then washed with phosphate-buffered saline and fixed in 3\% paraformaldehyde for 20 min at $4{ }^{\circ} \mathrm{C}$. Fixed cells were permeabilized with three washes with TBST (50 mM Tris-HCl, pH 7.4, $150 \mathrm{mM} \mathrm{NaCl,} \mathrm{0.1 \%} \mathrm{Tri-}$ ton X-100), and then blocked with 5.5\% HOS in TBST for $1 \mathrm{~h}$ at room temperature. Cells were then incubated with specific primary antibodies, diluted 1:100 in TBS (50 mM Tris-HCl, $\mathrm{pH} 7.4,150$ $\mathrm{mM} \mathrm{NaCl}$ ), overnight at $4{ }^{\circ} \mathrm{C}$. Cells were then washed once with TBST and once with TBST with $0.1 \%$ BSA and incubated with secondary antibodies (diluted 1:100) for $1 \mathrm{~h}$ at room temperature in TBST with $3 \%$ BSA. After extensive washes in TBST, cells were mounted with glycerol plastine and observed with a confocal fluorescence microscope (Leica TCS SP5).

\section{Differentiation and fusion indexes}

To determine the differentiation and fusion of $\mathrm{C} 2 \mathrm{C} 12$ myoblasts after specific treatment, we calculated the differentiation index as the percentage of mMHC-positive cells above the total nuclei and the fusion index as the average number of nuclei in mMHCpositive cells containing at least three nuclei above the total nuclei.

\section{Intracellular $\mathrm{H}_{2} \mathrm{O}_{2}$ assay}

Differentiating $\mathrm{C} 2 \mathrm{C} 12$ cells were stimulated with gAd and 3 min before the end of stimulation $5 \mu \mathrm{M}$ DCF-DA was added to the plate. Cells were then lysed in $1 \mathrm{ml}$ of RIPA buffer containing $1 \%$ Triton X-100 and fluorescence was analysed immediately using a Perkin-Elmer Fluorescence Spectrophotometer (excitation wavelength $488 \mathrm{~nm}$, emission wavelength $510 \mathrm{~nm}$ ). The values of fluorescence were normalized on total protein content.

\section{Glucose up-take assay}

To determine glucose uptake, $\mathrm{C} 2 \mathrm{C} 12$ were seeded in six-well plates, pre-treated with $\operatorname{HOS} 2 \%$ for $72 \mathrm{~h}$ and then stimulated with gAd $(1 \mu \mathrm{g} / \mathrm{ml})$. Subsequently, glucose transport was assayed essentially as described previously [11].

\section{RNA extraction}

$1 \times 10^{6}$ cells were pelletted and resuspended in $350 \mu$ of RLT buffer (Qiagen) and $10 \mu \mathrm{l} / \mathrm{ml} \beta$-mercaptoethanol. Total RNA from the cells was extracted with the RNeasy Mini reagent kit (Qiagen) according to the manufacturer's recommendations.

\section{Real time RT-PCR}

Total RNA (400 ng) was reverse transcribed using TaqMan Reverse Transcription Reagents Kit. Reverse transcription was performed in a final volume of $80 \mu$ containing $500 \mathrm{mM} \mathrm{KCl}, 0.1$ mM EDTA, $100 \mathrm{mM}$ Tris- $\mathrm{HCl}(\mathrm{pH} 8.3), 5.5 \mathrm{mM} \mathrm{MgCl} 2,500 \mu \mathrm{M}$ of each dNTP, $2.5 \mu \mathrm{M}$ random examers, $0.4 \mathrm{U} / \mu \mathrm{l}$ RNase inhibi- 
tor and $1.25 \mathrm{U} / \mu 1$ Multiscribe Reverse Transcriptase. The reverse transcription reaction was performed at $25{ }^{\circ} \mathrm{C}$ for $10 \mathrm{~min}, 48{ }^{\circ} \mathrm{C}$ for $30 \mathrm{~min}$ and $95{ }^{\circ} \mathrm{C}$ for $3 \mathrm{~min}$. Measurement of gene expression was performed by quantitative real-time PCR (ABI PRISM 7700 Sequence Detector, Applied Biosystems). The amount of target, normalized to an endogenous reference (eukaryotic 18S RNA, endogenous control, Applied Biosystems), was given by $2^{-\Delta \Delta C \mathrm{~T}}$ calculation [36]. For each sample, $12.5 \mathrm{ng}$ of cDNA was added to $10 \mu \mathrm{l}$ of PCR mix containing each primer/probe mix and $1 \times$ Universal Master Mix. The samples were then subjected to 40 cycles of amplification at $95{ }^{\circ} \mathrm{C}$ for $15 \mathrm{~s}$ and $60{ }^{\circ} \mathrm{C}$ for $60 \mathrm{~s}$. TaqMan Reverse Transcription Reagents Kit, all primer/probe mixes, Taqman Gene Expression Assays: Adipo R1 (ID number Mm01291331 m1) and Adipo R2 (ID number Mm01184032 m1), and 1× Universal Master Mix were from Applied Biosystems.

\section{Acknowledgments}

This work was supported by the Tuscany Region Studies on Rosiglitazone (TRESOR), the Italian Association for Cancer Research (AIRC), the Consorzio Interuniversitario Biotecnologie and the Cassa di Risparmio di Firenze.

\section{References}

1 Pajvani UB, Du X, Combs TP, et al. Structure-function studies of the adipocyte-secreted hormone Acrp30/adiponectin. Implications for metabolic regulation and bioactivity. $\mathrm{J}$ Biol Chem 2003; 278:9073-9085.

2 Berg AH, Combs TP, Scherer PE. ACRP30/adiponectin: an adipokine regulating glucose and lipid metabolism. Trends Endocrinol Metab 2002; 13:84-89.

3 Fruebis J, Tsao TS, Javorschi S, et al. Proteolytic cleavage product of 30-kDa adipocyte complement-related protein increases fatty acid oxidation in muscle and causes weight loss in mice. Proc Natl Acad Sci USA 2001; 98:2005-2010.

4 Kadowaki T, Yamauchi T. Adiponectin and adiponectin receptors. Endocr Rev 2005; 26:439-451.

5 Yamauchi T, Kamon J, Waki H, et al. The fat-derived hormone adiponectin reverses insulin resistance associated with both lipoatrophy and obesity. Nat Med 2001; 7:941-946.

6 Yamauchi T, Kamon J, Ito Y, et al. Cloning of adiponectin receptors that mediate antidiabetic metabolic effects. Nature 2003; 423:762-769.

7 Yamauchi T, Nio Y, Maki T, et al. Targeted disruption of AdipoR1 and AdipoR2 causes abrogation of adiponectin binding and metabolic actions. Nat Med 2007; 13:332-339.

8 Combs TP, Berg AH, Obici S, Scherer PE, Rossetti L. Endogenous glucose production is inhibited by the adipose-derived protein Acrp30. J Clin Invest 2001; 108:1875-1881.

9 Fiaschi T, Buricchi F, Cozzi G, et al. Redox-dependent and ligand-independent trans-activation of insulin receptor by globular adiponectin. Hepatology 2007; 46:130-139.

10 Yamauchi T, Kamon J, Minokoshi, Y et al. Adiponectin stimulates glucose utilization and fatty-acid oxidation by activating AMP-activated protein kinase. Nat Med 2002; 8:1288-1295.

11 Mao X, Kikani CK, Riojas RA, et al. APPL1 binds to adiponectin receptors and mediates adiponectin signalling and function. Nat Cell Biol 2006; 8:516-523.
12 Kadowaki T, Yamauchi T, Kubota N, Hara K, Ueki K, Tobe K. Adiponectin and adiponectin receptors in insulin resistance, diabetes, and the metabolic syndrome. J Clin Invest 2006; 116:1784-1792.

13 Blau HM, Pavlath GK, Hardeman EC, et al. Plasticity of the differentiated state. Science 1985; 230:758-766.

14 Charge SB, Rudnicki MA. Cellular and molecular regulation of muscle regeneration. Physiol Rev 2004; 84:209-238.

15 Molkentin JD, Olson EN. Defining the regulatory networks for muscle development. Curr Opin Genet Dev 1996; 6:445-453.

16 Bach LA, Salemi R, Leeding KS. Roles of insulin-like growth factor (IGF) receptors and IGF-binding proteins in IGF-II-induced proliferation and differentiation of L6A1 rat myoblasts. Endocrinology 1995; 136:5061-5069.

17 Ewton DZ, Roof SL, Magri KA, McWade FJ, Florini JR. IGFII is more active than IGF-I in stimulating L6A1 myogenesis: greater mitogenic actions of IGF-I delay differentiation. $J$ Cell Physiol 1994; 161:277-284.

18 Florini JR, Ewton DZ, Magri KA, Mangiacapra FJ. IGFs and muscle differentiation. Adv Exp Med Biol 1993; 343:319-326.

19 Conejo R, Valverde AM, Benito M, Lorenzo M. Insulin produces myogenesis in $\mathrm{C} 2 \mathrm{C} 12$ myoblasts by induction of NFkappaB and downregulation of AP-1 activities. J Cell Physiol 2001; 186:82-94.

20 Coolican SA, Samuel DS, Ewton DZ, McWade FJ, Florini JR. The mitogenic and myogenic actions of insulin-like growth factors utilize distinct signaling pathways. J Biol Chem 1997; 272:6653-6662.

21 Donati C, Meacci E, Nuti F, Becciolini L, Farnararo M, Bruni P. Sphingosine 1-phosphate regulates myogenic differentiation: a major role for S1P2 receptor. FASEB J 2005; 19:449-451.

22 Filigheddu N, Gnocchi VF, Coscia M, et al. Ghrelin and desacyl ghrelin promote differentiation and fusion of $\mathrm{C} 2 \mathrm{C} 12$ skeletal muscle cells. Mol Biol Cell 2007; 18:986-994.

23 Li Y, Jiang B, Ensign WY, Vogt PK, Han J. Myogenic differentiation requires signalling through both phosphatidylinositol 3-kinase and p38 MAP kinase. Cell Signal 2000; 12:751-757.

24 Yamauchi T, Kamon J, Waki H, et al. The fat-derived hormone adiponectin reverses insulin resistance associated with both lipoatrophy and obesity. Nat Med 2001; 7:941-946.

25 Furtado LM, Somwar R, Sweeney G, Niu W, Klip A. Activation of the glucose transporter GLUT4 by insulin. Biochem Cell Biol 2002; 80:569-578.

26 Delaigle AM, Jonas JC, Bauche IB, Cornu O, Brichard SM. Induction of adiponectin in skeletal muscle by inflammatory cytokines: in vivo and in vitro studies. Endocrinology 2004; 145:5589-5597.

27 Buckingham M. Myogenic progenitor cells and skeletal myogenesis in vertebrates. Curr Opin Genet Dev 2006; 16:525532.

28 Armand AS, Laziz I, Chanoine C. FGF6 in myogenesis. Biochim Biophys Acta 2006; 1763:773-778.

29 Floss T, Arnold HH, Braun T. A role for FGF-6 in skeletal muscle regeneration. Genes Dev 1997; 11:2040-2051.

30 Andres V, Walsh K. Myogenin expression, cell cycle withdrawal, and phenotypic differentiation are temporally separable events that precede cell fusion upon myogenesis. J Cell Biol 1996; 132:657-666.

31 Heron-Milhavet L, Mamaeva D, Rochat A, Lamb NJ, Fernan- 
dez A. Akt2 is implicated in skeletal muscle differentiation and specifically binds Prohibitin2/REA. J Cell Physiol 2008; 214:158-165.

$32 \mathrm{Wu} \mathrm{Z}$, Woodring PJ, Bhakta KS, et al. p38 and extracellular signal-regulated kinases regulate the myogenic program at multiple steps. Mol Cell Biol 2000; 20:3951-3964.

33 Long YC, Zierath JR. AMP-activated protein kinase signaling in metabolic regulation. J Clin Invest 2006; 116:1776-1783.

34 Waki H, Yamauchi T, Kamon J, et al. Generation of globular fragment of adiponectin by leukocyte elastase secreted by monocytic cell line THP-1. Endocrinology 2005; 146:790-796.

35 Pierce AP, de Waal E, McManus LM, Shireman PK, Chaudhuri AR. Oxidation and structural perturbation of redox-sensitive enzymes in injured skeletal muscle. Free Radic Biol Med 2007; 43:1584-1593.

36 Livak KJ, Schmittgen TD. Analysis of relative gene expression data using real-time quantitative PCR and the 2(-Delta Delta C(T)) method. Methods 2001; 25:402-408. 